\section{Personas mayores en Chile: el nuevo desafío social, económico y sanitario del Siglo XXI}

\author{
ANA MARÍA LEIVA ${ }^{1,2, a}$, \\ CLAUDIA TRONCOSO-PANTOJA ${ }^{3, b}$, \\ MARÍA ADELA MARTÍNEZ-SANGUINETTII ${ }^{4, c}$, \\ GABRIELA NAZAR ${ }^{5, \mathrm{~d}}$, \\ YENY CONCHA-CISTERNAS ${ }^{6,7, e}$, \\ MIQUEL MARTORELL ${ }^{8, \mathrm{f}}$, \\ KARINA RAMÍREZ-ALARCÓN ${ }^{8, \mathrm{~g}}$,

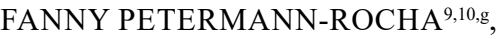 \\ IGOR CIGARROA ${ }^{7, \mathrm{~h}}$, XIMENA DÍAZ ${ }^{11, \mathrm{I}}$, \\ CARLOS CELIS-MORALES ${ }^{9,10,12,13, j}$, \\ en representación del consorcio de investigación \\ ELHOC-Chile (Epidemiology of Lifestyle and \\ Health Outcomes in Chile).
}

\section{Older people in Chile: the new social, economic and health challenge for the $21^{\text {st }}$ century}

Ageing will be one of the most significant social transformations of the $21^{\text {st }}$ century worldwide. In the last 40 years, Chile has tripled its older adult population. As a result, by 2050 the country will have the highest proportion of older adults in Latin America. This remarkable growth reinforces the need to identify their current situation and to revise what is the society doing to maintain older people as active members. In this context, this narrative revision aimed to describe the sociodemographic, epidemiologic and sociocultural profile of the older Chilean adults. Besides, programs and public policies focused on the improvement of their quality of life were identified.

(Rev Med Chile 2020; 148: 799-809)

Key words: Aging; Public Policy; Quality of Life; Chile.
'Instituto de Anatomía, Histología y Patología, Facultad de Medicina, Universidad Austral de Chile. Valdivia, Chile. ${ }^{2}$ Doctorado en Investigación Gerontológica. Universidad Maimónides. Buenos Aires Ciudad Autónoma, Argentina. ${ }^{3}$ CIEDE-UCSC, Departamento de Salud Pública, Facultad de Medicina, Universidad Católica de la Santísima Concepción. Concepción, Chile.

${ }^{4}$ Instituto de Farmacia, Facultad de Ciencias, Universidad Austral de Chile. Valdivia, Chile.

${ }^{5}$ Facultad de Ciencias Sociales, Centro Vida Saludable Universidad de Concepción. Concepción, Chile.

${ }^{6}$ Pedagogía en Educación Física, Facultad de Educación, Universidad Autónoma de Chile, Chile.

${ }^{7}$ Escuela de Kinesiología, Facultad de Salud, Universidad Santo Tomás. Chile.

${ }^{8}$ Departamento de Nutrición y Dietética, Facultad de Farmacia, Universidad de Concepción. Concepción, Chile.

IInstitute of Health and Wellbeing, University of Glasgow, Glasgow, UK.

${ }^{10} \mathrm{BHF}$ Glasgow Cardiovascular Research Centre, Institute of Cardiovascular and Medical Sciences, University of Glasgow, Glasgow, UK.

"Grupo de Calidad de Vida, Departamento de Ciencias de la Educación, Universidad del Bio-Bío. Chillán, Chile.

${ }^{12}$ Centro de Investigación en Fisiología del Ejercicio-CIFE, Universidad Mayor. Santiago, Chile.

${ }^{13}$ Laboratorio de Rendimiento Humano, Grupo de Estudio en Educación, Actividad Física y Salud (GEEAFyS),

Universidad Católica del Maule. Talca, Chile.

aProfesor de Biología y Química. MSc. Neurociencias y

Salud Mental.

${ }^{b}$ Nutricionista, MSc. Salud Pública basada en la evidencia.

MSc. en Gerontología.

'Bioquímico, MSc Nutrición y Dietética.

dPsicólogo. Doctor en Humanidades y Ciencias Sociales.

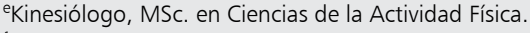

fBioquímico, Doctor en Nutrición Humana.

${ }^{9}$ Nutricionista, MSc. Nutrición Humana.

hKinesiólogo, Doctor en Neurociencias.

'Profesor de Educación Física. MSc Educación Física. Doctor en Innovación Didáctica y Formación del Profesorado.

iProfesor de Educación Física, Doctor Ciencias

Cardiovasculares y Biomédicas.

Trabajo no recibió financiamento.

Los autores declaran no tener conflictos de interés.

Recibido el 4 de julio de 2019, aceptado el 8 de junio de 2020

Correspondencia a:

Ana María Leiva Ordoñez

Instituto de Anatomía, Histología y Patología,

Facultad de Medicina,

Universidad Austral de Chile. Valdivia, Chile.

aleiva@uach.cl

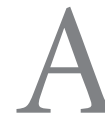

nivel mundial, el envejecimiento de la población será una de las transformaciones sociales más relevantes del siglo XXI, proyectándose que el número de personas mayores (PM) se duplicará para el 2050 y triplicará para el $2100^{1}$.
Chile es unos los países latinoamericanos que ha experimentado un acelerado incremento de su población adulta mayor, esperándose que hacia el 2040 , sobre $20 \%$ de la población supere los 60 años y $6 \%$ alcance los 80 años $^{2,3}$. Esta realidad exige un 
urgente replanteamiento de las políticas públicas que permitan dar respuestas a este escenario de envejecimiento poblacional ${ }^{4-6}$. El Plan de acción mundial sobre el envejecimiento y salud de la Organización Mundial de la Salud (OMS) señala la necesidad de generar evidencia para la elaboración de políticas públicas enfocadas en este grupo de la población ${ }^{7}$. En consecuencia, el objetivo de esta revisión narrativa fue describir el perfil sociodemográfico, epidemiológico y sociocultural de las personas mayores en Chile, identificando programas y políticas públicas orientadas a mejorar su calidad de vida.

\section{Materiales y Métodos}

\section{Fuentes de información y estrategia de búsqueda}

Revisión narrativa generada a partir de buscadores Web of Science, Medline, Scopus, Scielo y Google Scholar y buscadores específicos de los Ministerios de Salud y Desarrollo Social de Chile, bases de datos de la Organización Mundial de la Salud (OMS) y Organización Panamericana de la Salud (OPS). Las palabras claves utilizadas en la búsqueda fueron: envejecimiento, adulto mayor y persona mayor; integrando los conceptos: políticas públicas, desafíos, estadísticas, prioridades, reporte, encuesta, guías y recomendaciones. Los criterios de inclusión fueron: (i) personas mayores en Chile (> 60 años); (ii) informes y reportes de entidades de salud nacionales e internacionales; (iii) informes y reportes de organismos guberna- mentales y de centros de estudios. Los estudios o reportes que no cumplieran con estos criterios fueron excluidos. La búsqueda incluyó publicaciones entre los años 2002 y 2019, considerando el año de inicio de la implementación de la política de envejecimiento; ampliando la búsqueda con palabras claves en inglés para incluir las recomendaciones internacionales.

\section{Antecedentes}

\section{Perfil sociodemográfico de las personas mayores}

Las mejoras en las condiciones de salud y disminución de las tasas de mortalidad y fecundidad han desencadenado un cambio sociodemográfico global, donde, por primera vez, las personas pueden aspirar a vivir más allá de los 60 años. Chile no está ajeno a esta realidad, al ser el único país latinoamericano que proyecta para el 2050 tener más de $30 \%$ de su población con 60 años o más, estimándose que entre los años 2015 y 2050, el incremento de las PM será de 109,5\%; superando el $74,7 \%$ proyectado para la población mundial ${ }^{8,9}$ (Figura 1). El aumento en 11,4 años en el promedio de edad de la población ${ }^{10}$ (Figura 2) implicará que Chile será el único país de la región de las Américas que tendrá una esperanza de vida media que superará los 80 años ${ }^{11}$.

El 40,1\% de los hogares chilenos tiene un adulto mayor entre sus integrantes, lo que representa $19,3 \%$ de la población total ${ }^{12}$. Respecto a su lugar de residencia, $85 \%$ vive en la ciudad, lo que implica

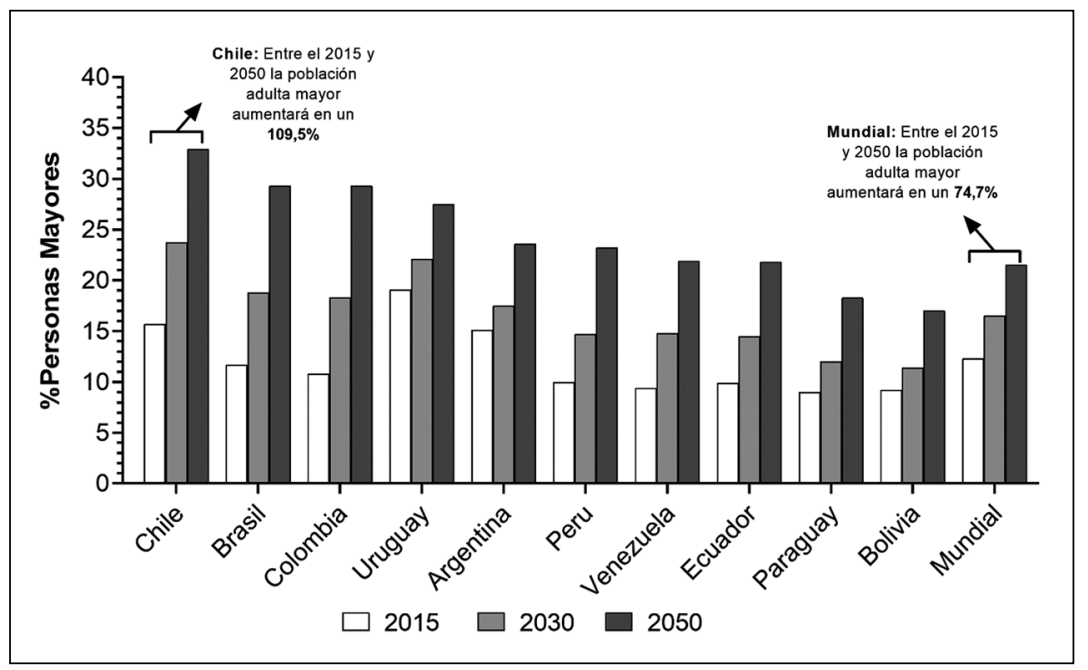

Figura 1. Proyecciones de la prevalencia de personas mayores en la región latinoamericana. Datos presentados como porcentaje de personas mayores a 60 años según países. Datos extraídos y adaptados del reporte de las Naciones Unidas ${ }^{10}$. 
que las ciudades deberán ser rediseñadas, contando con infraestructura y servicios que faciliten su desplazamiento y autonomía ${ }^{13}$.

El 7,9\% de las PM está bajo la línea de la pobreza, señalando que sus ingresos no les alcanzan para cubrir la canasta básica alimentaria y no alimentaria ${ }^{14}$. En Chile, a partir de 2010, la pobreza se
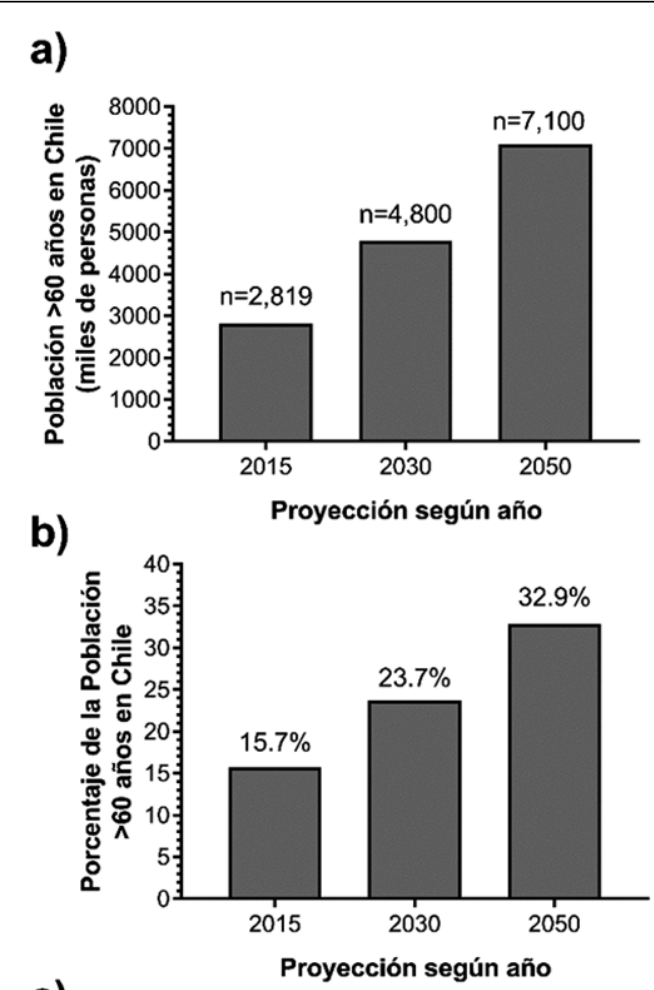

c)

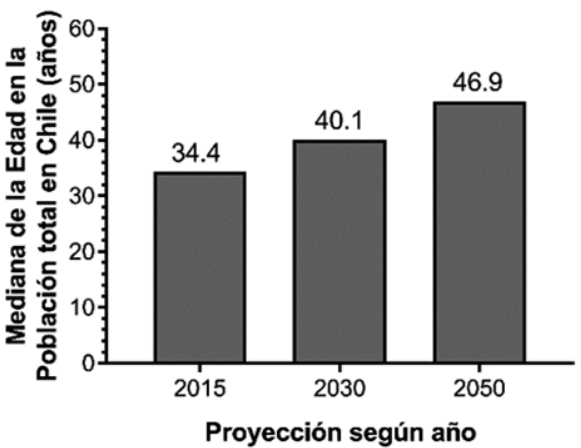

Figura 2. Proyecciones de personas mayores en Chile en las próximas 3 décadas. Datos presentados como: a) cantidad de personas mayores expresadas en miles de personas; b) porcentaje de personas mayores a 60 años y c) mediana de la edad en Chile. Datos extraídos y adaptados del Reporte de las Naciones Unidas ${ }^{10}$. mide con el "Índice de Pobreza Multidimensional" (PMD), que, además de los ingresos económicos, considera otros aspectos asociados a las condiciones de vida de las personas ${ }^{15,16}$. Considerando la relación inversa entre nivel educacional y PMD, aunque la escolaridad de las PM ha aumentado significativamente desde 1990 a $2015^{17}$, actualmente, tres de cada cuatro adultos mayores no tienen educación media, lo que impone un desafío país, para superar este indicador determinante de la calidad de vida ${ }^{16,18}$.

\section{Perfil de morbimortalidad y factores de riesgo}

El estudio de Carga Global de Enfermedad, realizado en 195 países entre los años 1990 y 2017, determinó que de 293 enfermedades registradas en el sistema de Clasificación Internacional de Enfermedades (ICD10), 92 estaban directamente vinculadas al envejecimiento ${ }^{19,20}$. A nivel mundial, los años de vida perdidos por discapacidad, enfermedad o mortalidad prematura atribuibles al envejecimiento (DALYs), corresponde a 138,7 DALYs por cada 1.000 adultos, cifra muy similar a Chile, que si bien está dentro de los 3 países con menor DALYs en Latinoamérica, aún está lejos de los países con los mejores niveles de envejecimiento saludable en el mundo (Figura 3$)^{20}$. Las PM en Chile presentan coexistencia de 2 o más enfermedades crónicas (multimorbilidad) (Figura 4) ${ }^{19,21}$; condición que incrementa la demanda de cuidados a largo plazo y costos de salud asociados ${ }^{22,23}$. El segundo estudio nacional de dependencia en población adulta mostró que 38,3\% de las PM en Chile tiene dependencia en cualquiera de sus grados ${ }^{24}$, lo que evidencia la necesidad de implementar un sistema de cuidados a largo plazo ${ }^{25}$. Un avance es el Programa de Larga Estadía para Adultos Mayores (ELEAM), orientado a mejorar las condiciones de vida de las PM dependientes y vulnerables que residen en este tipo de establecimientos ${ }^{26}$. Dado que el estado funcional es el mayor determinante de calidad de vida de las PM, prevenir y revertir la dependencia debe ser prioritario en la agenda pública de Chile ${ }^{18}$.

Otra condición asociada al envejecimiento es la fragilidad; las personas frágiles, según los criterios de Fried, presentan pérdida involuntaria de peso corporal, sentimiento de agotamiento general, debilidad, lentitud al caminar y bajos niveles de actividad física ${ }^{23,27}$. El síndrome de fragilidad está presente en $13,2 \%$ de la población mayor de 60 


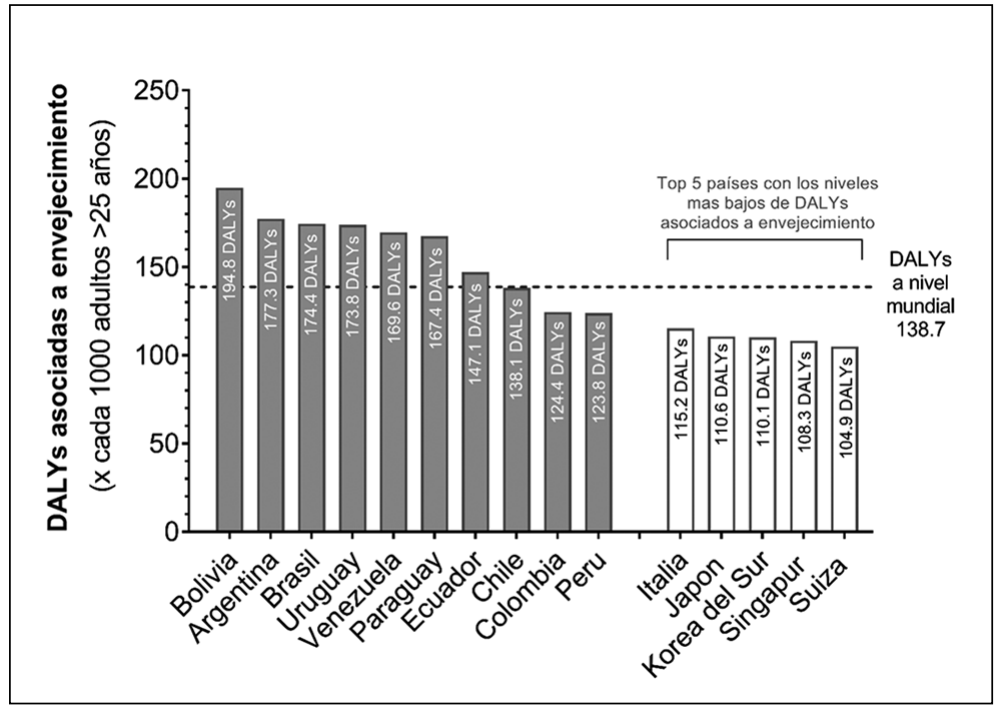

Figura 3. DALYs asociados a envejecimiento, comparación de países de Latinoamérica con países con los niveles más bajos de DALYs a nivel mundial ${ }^{20}$.

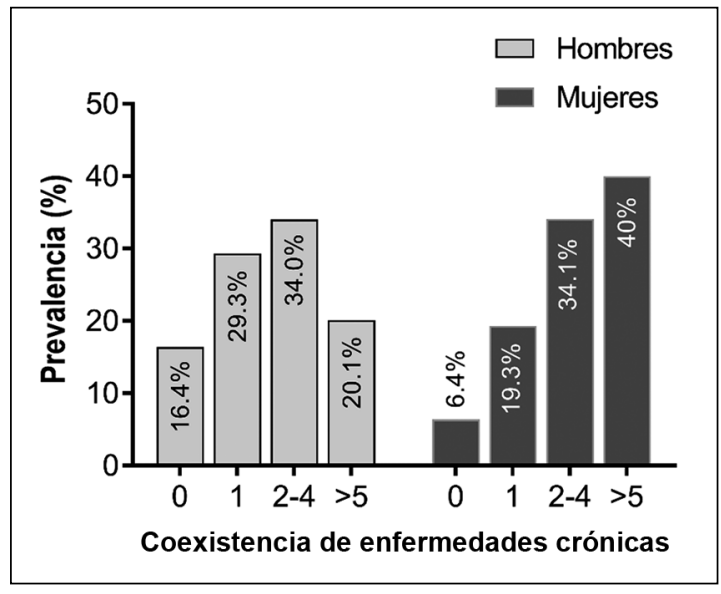

Figura 4. Prevalencia de multimorbilidad en personas mayores en Chile. Datos presentados como prevalencia expandida a población nacional. Multimorbilidad fue determinada en base a 14 enfermedades crónicas (diabetes mellitus, hipertensión arterial, infarto de miocardio, accidente cerebrovascular, enfermedad vascular periférica, depresión, cáncer, osteoporosis, insuficiencia renal, EPOC, asma, apnea del sueño, colesterol elevado). Datos extraídos de la Encuesta Nacional de Salud 2016-201721.

años en Chile, siendo superior en mujeres (20,0\%) que en hombres $(6,1 \%)$ y su prevalencia aumenta con la edad (Figura 5) ${ }^{21,28}$. Con el envejecimiento aumenta también la prevalencia de Alzheimer y otros tipos de demencias. En el año 2016 en Chile,
104.523 personas presentaron Alzheimer u otras formas de demencia, siendo 70.944 DALYs atribuidos como carga a esta enfermedad ${ }^{23}$.

En cuanto a la mortalidad, en 2017 ocurrieron 77.318 defunciones de PM, representando 72,7\% del total de defunciones ocurridas en el país, con una tasa de 37,3 por cada mil personas de 65 años o más. Del total de muertes, $31,0 \%$ correspondieron a enfermedades del sistema circulatorio, seguido de $25,1 \%$ debido a tumores malignos ${ }^{29}$.

\section{Necesidades de las personas mayores}

El crecimiento exponencial de las PM exige un mayor conocimiento de cómo este segmento de la población puede experimentar un envejecimiento exitoso, lo que está directamente relacionado con la satisfacción de sus necesidades básicas, como acceso íntegro a la salud, seguridad económica y participación social ${ }^{30,31}$.

\section{Acceso íntegro a la salud}

La salud es una necesidad prioritaria, al impactar directamente en la calidad de vida y funcionalidad de las $\mathrm{PM}^{32,33}$. En Chile, $87 \%$ de la población adulta mayor se atiende en el sistema de salud público (FONASA) ${ }^{34}$, por lo que las acciones desarrolladas desde este ámbito tienen un impacto significativo en esta población. El Ministerio de Salud (MINSAL) ha implementado varias prestaciones que van en directo beneficio de 


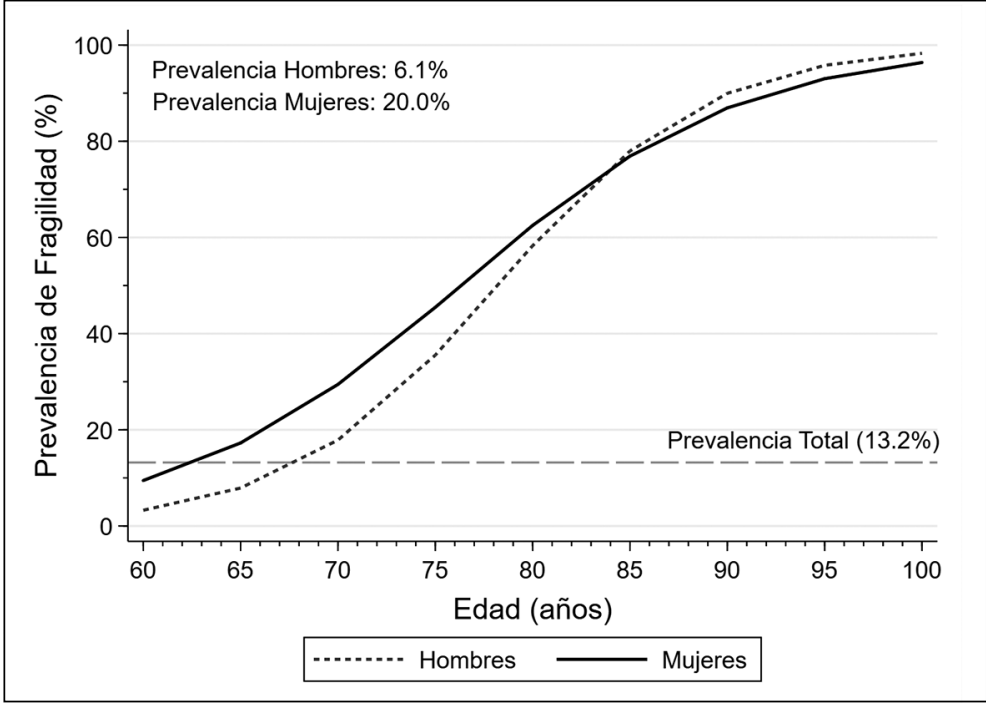

Figura 5. Prevalencia de fragilidad en personas mayores, según edad y sexo en Chile. Datos presentados como prevalencia expandida a población nacional según sexo. Fragilidad fue definida como la presencia de al menos 3 de los siguientes criterios: pérdida involuntaria de peso corporal, bajos niveles de actividad física, debilidad muscular, inhabilidad de caminar a un paso rápido o normal y que reporten cansancio o fatiga al realizar tareas de la vida cotidiana. Resultados derivados de la Encuesta Nacional de Salud 2016-201721. las PM; encontrándose entre las prestaciones de salud de alto impacto, la eliminación del copago para personas de 65 años y más ${ }^{26}$. También está el programa "Más Adultos Mayores Autovalentes" orientado a mejorar o mantener la funcionalidad de las PM y el Examen de Medicina Preventiva del Adulto Mayor (EMPAM), orientado a detectar en forma oportuna factores de riesgos. Este incluye el Examen de Funcionalidad del Adulto Mayor (EFAM) que permite clasificar grado de funcionalidad ${ }^{35}$. Otra prestación es el programa de Garantías Explícitas en Salud (GES), que además de asegurar el tratamiento de ciertas patologías a toda la población, cubre algunas patologías de alta prevalencia ${ }^{37}$. Una de las debilidades de estos programas es su baja cobertura y escasa evaluación de sus procesos y resultados sanitarios ${ }^{36-38}$.

Otra necesidad básica de las PM, determinante del envejecimiento exitoso, es la alimentación. De acuerdo con la Encuesta Calidad de Vida en la Vejez, la alimentación es una de las necesidades mayormente satisfechas en las PM (89\% de satisfacción $)^{39}$. Un beneficio en este ámbito es el Programa de Alimentación Complementaria del Adulto Mayor (PACAM), que entrega alimentos fortificados con micronutrientes, para mantener y mejorar su estado nutricional ${ }^{37}$. En Chile, tres de cada cuatro PM tienen una dieta balanceada, es decir, que incorpora legumbres, pescado, frutas y verduras. El 90\% consume porotos, lentejas o garbanzos una vez por semana; 71,9\% consume pescado al menos una vez por semana y $71,6 \%$ consume verduras o frutas, al menos 5 porciones al día ${ }^{39}$.

\section{Seguridad económica y protección social}

El envejecimiento saludable está influenciado significativamente por los determinantes sociales de la salud; evidenciándose que las personas de niveles socioeconómicos bajos tienen peor salud y menor esperanza de vida ${ }^{40}$.

Los ingresos de las PM en Chile provienen fundamentalmente de las pensiones, declarando un tercio de ellos no lograr cubrir sus necesidades básicas $^{41}$. El 54,4\% no recibe pensión ${ }^{37}$ y 37,9\% depende del aporte del Pilar Solidario, beneficio del Estado que apoya con pensiones básicas a las PM más vulnerables ${ }^{14,42}$.

Está demostrado que los países que cuentan con mayor inversión en seguridad social tienen menor nivel de pobreza. En Latinoamérica, los países que registran mayor nivel de cobertura en seguridad social son Uruguay, Argentina, Brasil y Chile? . Esta información es concordante con los resultados de la Encuesta de Calidad de Vida del Adulto Mayor, que señala que $77 \%$ de las PM están satisfechas con su vida, a pesar que la dimensión económica es una de las necesidades menos satisfechas ${ }^{43}$, lo que podría interpretarse con la valoración de otros ámbitos de la vida. 


\section{Participación social}

La participación social es otra de las necesidades relevantes para las $\mathrm{PM}$, al promover su bienestar y la calidad de vida, constatándose incluso que las PM que participan en organizaciones comunitarias tienen mejor desempeño en actividades funcionales y mayor satisfacción con su vida ${ }^{43-45}$.

La participación social es considerada un recurso psicosocial determinante del envejecimiento exitoso. En Chile, en el marco de la política de envejecimiento, se han implementado varios programas, evidenciándose un aumento sistemático de la participación social de las PM en distintos ámbitos de la sociedad ${ }^{41,46,47}$ (Tablas 1 y 2). Una forma de participación social es el trabajo, que incide positivamente en la salud y bienestar de las PM. Actualmente, la tasa de empleo de las PM entre 60 y 64 años es de 62,2\%; porcentaje que desciende a 37,1\% en el tramo de 65-69 años y a $24,2 \%$ en el grupo de 70-79 años ${ }^{48}$.

\section{Cuidados de largo plazo en el contexto}

del envejecimiento poblacional

El perfil de envejecimiento de la población chilena, y la estrecha relación entre el envejecimiento y la pérdida de autonomía, demandará importantes desafíos en término de políticas públicas, como los cuidados a largo plazo (CLP) ${ }^{6}$. En Chile, los programas en torno a esta necesidad son de baja cobertura, solo $5 \%$ de las PM con dependencia se encuentran en casas de cuidados ${ }^{17}$. Es más, el programa más grande en Chile que entrega servicios de CLP solo cubre a $11 \%$ de la población usuaria que lo necesita ${ }^{17}$. En Chile, la cobertura y disponibilidad de residencias de PM tienden a concentrarse en municipios con mayor población y mayores ingresos. Considerando la alta demanda que existirá por parte de PM con grado de dependencia, la implementación de un sistema de CLP y su financiamiento debe ser prioridad en la agenda pública del país ${ }^{25}$.

\section{Carga de los cuidados informales e inequidad de} género

La dependencia en PM no se distribuye en forma igualitaria, manteniendo grandes diferencias de prevalencia urbano-rural, en quienes son afiliados al sistema público de salud y mujeres de mayor edad ${ }^{49}$.

Según el Informe de Desarrollo Social de 2017, $95 \%$ de los cuidadores eran mujeres, para quienes el costo del cuidado implica tiempo, oportunidades perdidas de generar ingresos, pérdida de contribuciones previsionales, por no participación laboral o abandono anticipado del mercado del trabajo. Los cuidadores, en general, tienen menor esperanza de vida, porque asumen una enorme carga física y psicológica que permanece invisible a ojos de la sociedad, al no ser considerada por el sistema de seguridad social ${ }^{24}$.

\section{Política Integral de Envejecimiento en Chile y sus desafíos}

Para enfrentar el envejecimiento poblacional con éxito, es necesario desarrollar políticas públicas que aborden los factores que afectan la manera

Tabla 1. Principales programas de política de envejecimiento positivo en Chile. Eje saludable*

\begin{tabular}{|c|c|c|}
\hline Programas (responsable) & Objetivo & Beneficiarios \\
\hline "Centro Día" (Municipios) & $\begin{array}{l}\text { Entregar prestaciones de servicios socio-sanita- } \\
\text { rios y apoyo familiar preventivo, atendiendo a } \\
\text { las necesidades personales básicas, actividades } \\
\text { socio-culturales y de promoción para un enve- } \\
\text { jecimiento activo, favoreciendo la autonomía }\end{array}$ & $\begin{array}{l}\text { Personas mayores de } 60 \text { años, de los tres } \\
\text { primeros quintiles de vulnerabilidad, con } \\
\text { dependencia leve, deterioro cognitivo } \\
\text { leve o depresión leve }\end{array}$ \\
\hline $\begin{array}{l}\text { "Cuidados domiciliarios" } \\
\text { (Municipios) }\end{array}$ & $\begin{array}{l}\text { Mejorar la calidad de vida de las personas } \\
\text { mayores en situación de vulnerabilidad y de- } \\
\text { pendencia }\end{array}$ & $\begin{array}{l}\text { Personas mayores de } 60 \text { años, de los tres } \\
\text { primeros quintiles de vulnerabilidad, con } \\
\text { dependencia moderada o grave }\end{array}$ \\
\hline $\begin{array}{l}\text { "Establecimientos de Larga } \\
\text { Estadía" (SENAMA) }\end{array}$ & $\begin{array}{l}\text { Contribuir a que las personas mayores accedan } \\
\text { a servicios integrales de apoyo y cuidados de } \\
\text { acuerdo con sus niveles de dependencia }\end{array}$ & $\begin{array}{l}\text { Personas mayores de } 60 \text { años, de los tres } \\
\text { primeros quintiles de vulnerabilidad, con } \\
\text { dependencia moderada o grave }\end{array}$ \\
\hline
\end{tabular}

*Información basada en el documento "Balance de Gestión Integral 2018", del Ministerio de Desarrollo Social, Servicio Nacional del Adulto Mayor (SENAMA) ${ }^{54}$. 
Tabla 2. Principales programas de política de envejecimiento positivo en Chile. Eje Participación*

\begin{tabular}{|c|c|c|}
\hline Programa responsable & Objetivo & Beneficiarios \\
\hline $\begin{array}{l}\text { "Buen Trato" } \\
\text { SENAMA }\end{array}$ & $\begin{array}{l}\text { Contribuir a la promoción y al ejercicio de los derechos de } \\
\text { las personas mayores a través de la prevención, protección y } \\
\text { promoción del buen trato }\end{array}$ & Personas mayores de 60 años \\
\hline $\begin{array}{l}\text { "Ciudades Amigables" } \\
\text { (SENAMA, en colaboración } \\
\text { con municipios) }\end{array}$ & $\begin{array}{l}\text { Promover ciudades amigables con las personas mayores, } \\
\text { abordando los desafíos urbanos y rurales propios de una } \\
\text { población envejecida, con el fin de fomentar la autonomía, } \\
\text { participación y calidad de vida de este grupo etario }\end{array}$ & Personas mayores de 60 años \\
\hline $\begin{array}{l}\text { "Turismo Social" } \\
\text { (Municipios) }\end{array}$ & $\begin{array}{l}\text { Brindar oportunidad de acceso a espacios de recreación y es- } \\
\text { parcimiento para adultos mayores vulnerables que no tienen } \\
\text { acceso a satisfacer esta necesidad por sus propios medios }\end{array}$ & $\begin{array}{l}\text { Personas mayores, de organi- } \\
\text { zaciones de adultos mayores } \\
\text { o programas de beneficencia }\end{array}$ \\
\hline $\begin{array}{l}\text { "Envejecimiento } \\
\text { Activo" } \\
\text { (SENAMA) }\end{array}$ & $\begin{array}{l}\text { Lograr que los adultos mayores participen de actividades que } \\
\text { promuevan el envejecer activamente }\end{array}$ & $\begin{array}{l}\text { Personas mayores de } 60 \text { años, } \\
\text { autovalentes }\end{array}$ \\
\hline $\begin{array}{l}\text { "Asesores Seniors" } \\
\text { (SENAMA) }\end{array}$ & $\begin{array}{l}\text { Promover la integración social y relaciones intergeneracio- } \\
\text { nales de personas mayores, preferentemente profesionales } \\
\text { jubiladas }\end{array}$ & $\begin{array}{l}\text { Personas mayores de } 60 \text { años, } \\
\text { enseñanza media completa, } \\
\text { autovalentes }\end{array}$ \\
\hline $\begin{array}{l}\text { "Vínculos" } \\
\text { (SENAMA) }\end{array}$ & $\begin{array}{l}\text { Generar condiciones que permitan a los usuarios acceder en } \\
\text { forma preferente a las prestaciones sociales pertinentes a sus } \\
\text { necesidades e integrarse a la red comunitaria de protección } \\
\text { social }\end{array}$ & $\begin{array}{l}\text { Personas mayores de } 60 \text { años, } \\
\text { de los tres primeros quintiles } \\
\text { de vulnerabilidad }\end{array}$ \\
\hline $\begin{array}{l}\text { "Fondo Nacional del } \\
\text { Adulto Mayor" } \\
\text { (SENAMA) }\end{array}$ & $\begin{array}{l}\text { Financiar iniciativas de organizaciones públicas o privadas, } \\
\text { apoyando a personas mayores vulnerables, para promover, } \\
\text { mantener o mejorar su condición de vida }\end{array}$ & $\begin{array}{l}\text { Personas mayores de } 60 \text { años, } \\
\text { de organizaciones de adultos } \\
\text { mayores }\end{array}$ \\
\hline
\end{tabular}

*Información basada en el documento "Balance de Gestión Integral 2018", del Ministerio de Desarrollo Social, Servicio Nacional del Adulto Mayor (SENAMA) ${ }^{54}$.

de envejecer de las poblaciones ${ }^{50,51}$. En este contexto, en 2012, el SENAMA desarrolló la "Política Integral de Envejecimiento Positivo 2012-2025”, orientada a la creación de entornos favorables que contribuyan a una mejor calidad de vida $y$ favorezcan la autonomía e independencia de las $\mathrm{PM}^{51,52}$. Una política en este ámbito debe abordar de forma transversal el envejecimiento en todos los servicios del Estado. La política se centra en 4 ejes de trabajo: Saludable, Seguro, Participativo e Institucional, con líneas de acción intersectorial de responsabilidad de distintos organismos ${ }^{53,54}$. El eje central de la política es la protección social de las $\mathrm{PM}$, siendo este ámbito el que mayor desarrollo evidencia en cuanto a número de programas desarrollados ${ }^{55}$. Los programas están principalmente centralizados en el SENAMA, otros son ejecutados a través de los municipios u otras entidades gubernamentales ${ }^{13,37,45,46}$ (Tablas 1 y 2 ).

La Política Integral de Envejecimiento Positivo constituye un avance sustancial en materias de envejecimiento, reflejándose un aumento en la satisfacción vital ${ }^{2,41}$, sin embargo, aún existen deficiencias que superar, especialmente en términos de salud, economía y trabajo ${ }^{53}$, además de la falta de evaluación de los programas, su impacto en la calidad de vida de las PM, y cómo contribuyen al cumplimiento de los objetivos formulados en la política $^{38,53}$. Una gran deuda pendiente es la inversión en salud pública y el déficit de especialistas en geriatría. La recomendación internacional indica un especialista por cada 5.000 PM y en nuestro país la relación es de 1 por cada $60.000^{56}$. Al ser la salud el principal determinante de la calidad de vida de las poblaciones ${ }^{57,58}$, este componente de la política debería ser prioridad en los próximos años.

\section{Conclusión}

Las PM en Chile viven más, consecuentemente, presentan mayor prevalencia de enfermedades 
crónicas, con una gran carga de morbimortalidad y pérdida de años de vida ajustados por discapacidad. Han aumentado su nivel educacional, están más organizados y activos que generaciones pasadas y presentan mayor satisfacción con su vida. La valorización del envejecimiento, suscribiendo acuerdos internacionales y una política integral, con numerosos programas, ha contribuido a reforzar sus capacidades y responder a las necesidades de las PM. Una debilidad de la política es la falta de evaluación de los programas en términos de resultados sanitarios. La dimensión social de la política, en cuanto a número de programas, ha logrado un mayor desarrollo respecto la dimensión salud. En este sentido, el foco en los próximos años debe estar en acciones más integradas de todos los sectores, centradas en un modelo socio-comunitario que ponga el centro en la calidad de vida de las personas mayores.

\section{Referencias}

1. World Health Organization. Global Health and Aging. National Institute on Aging. National Institutes of Health. WHO, 2011. Disponible en: https://www.who. int/ageing/publications/global_health.pdf.

2. Asociación de Municipalidades de Chile (AMUCH). Los Adultos Mayores en las Comunas de Chile: Actualidad y Proyecciones. Abril 2017. Disponible en: http://www.amuch.cl/wp-content/uploads/2017/05/ ESTUDIO-ADULTO-MAYOR-EN-LAS-COMUNAS-DE-CHILE_-PROYECCIONES.pdf.

3. Instituto Nacional de Estadísticas. Chile. Adultos Mayores en Cifras. Estadísticas Vitales 2016. INE, 2018. Disponible en: https://www.ine.cl/docs/default-source/ publicaciones/2018/adultos-mayores-cifras-vitales. pdf?sfvrsn=1eff56d2_6.

4. Ogura S, Jakovljevic M. Global Population Aging-Health Care, Social and Economic Consequences. Front Public Health 2018; 6: 335.

5. Centro UC Políticas Públicas. Asociación AFP Chile. Adultos Mayores: un activo para Chile. Santiago, Chile, 2017. Disponible en: https://politicaspublicas.uc.cl/ wp-content/uploads/2017/06/Adultos-Mayores-un-activo-para-Chile.pdf.

6. Villalobos Dintrans P. Envejecimiento y cuidados a largo plazo en Chile: desafíos en el contexto de la OCDE. Rev Panam Salud Publica. 2017; 41: e86.

7. World Health Organization. Global strategy and action plan on ageing and health. WHO, 2017. Disponible en: https://www.who.int/ageing/WHO-GSAP-2017. pdf?ua $=1$.

8. World Health Organization. Multisectoral action for healthy aging based on the life cycle: draft global strategy and plan of action on aging and health: Report by the Secretariat. WHO, 2016. Disponible en: https://apps. who.int/iris/bitstream/handle/10665/252671/A69_17en.pdf? sequence $=1$ \&isAllowed $=y$.

9. Aranco N, Stampini M, Ibarrán P, Medellin. Panorama de envejecimiento y dependencia en América Latina y el Caribe. Banco Interamericano de Desarrollo. División de Protección Social y Salud. Resumen de Políticas $\mathrm{N}^{\circ}$ IDP-PB-273. BDI, enero de 2018. Disponible en: https://www.gerontologia.org/portal/archivosUpload/ uploadManual/Panorama-de-envejecimiento-y-dependencia-en-America-Latina-y-el-Caribe.PDF.

10. United Nations. Department of Economic and Social Affairs. Population Division. World Population Ageing, 2015. (ST/ESA/SER.A/390). Disponible en: https://www. un.org/en/development/desa/population/publications/ pdf/ageing/WPA2015_Report.pdf.

11. World Health Organization. World Health Statistics 2018. Monitoring health for the SDGs, Sustainable Development Goals. Geneva: WHO, 2018. Disponible en: https://apps.who.int/iris/handle/10665/272596.

12. Ministerio de Desarrollo Social. Gobierno de Chile. Pobreza y Distribución de Ingresos. Presentación de resultados. Encuesta CASEN 2017. Observatorio Social. 2018. Disponible en: http://observatorio.ministeriodesarrollosocial.gob.cl/casen-multidimensional/casen/docs/ Presentacion_Sintesis_de_Resultados_Casen_2017.pdf.

13. World Health Organization. Global Cities Friendly to the Elderly: A Guide. WHO, 2007. ISBN 979924 354730 5. Disponible en: https://www.who.int/ageing/ publications/Global_age_friendly_cities_Guide_English. pdf.

14. Ministerio de Desarrollo Social. Gobierno de Chile. Informe de Desarrollo Social 2018. Disponible en: http:// www.ministeriodesarrollosocial.gob.cl/storage/docs/ Informe_de_Desarrollo_Social_2018.pdf.

15. Berner Herrera H. La medición de pobreza multidimensional, mirada comprehensiva a la pobreza y desigualdad en Chile. Importancia de variables territoriales y sociales. Subsecretaría de Evaluación Social. Gobierno de Chile, 2016. Disponible en: http://www.desarrollosocialyfamilia.gob.cl/pdf/upload/Heidi_Berner_MDS. pdf

16. Cabieses B, Pedreros V, Oyarte M, Sirlopu D, Alfaro J, Bernales M, et al. Pobreza Multidimensional, Salud Auto-percibida y Acceso a Salud en Chile. Generando Conocimiento Crítico para la Acción en Red en Salud 
Pública. Universidad del Desarrollo, 2017. Disponible en: https://repositorio.udd.cl/handle/11447/1633.

17. Ministerio de Desarrollo Social. Subsecretaria de Evaluación Social. Encuesta de Caracterización Socioeconómica Nacional. CASEN 2015. 22 de septiembre, 2016. Disponible en: http://www.gobernacionlosandes. gob.cl/media/2016/09/Presentaci\%C3\%B3n-Casen-2015-5.pdf.

18. León D, Rojas M, Campos F. Guía Calidad de Vida en la Vejez. Herramientas para vivir más y mejor. Pontificia Universidad Católica de Chile. Vicerrectoría de Comunicaciones y Educación Continua Programa Adulto Mayor Centro de Geriatría y Gerontología. Santiago de Chile, 2011. Disponible en: http://adultomayor.uc.cl/ docs/guia_calidad_de_vida.pdf.

19. Ministerio de Salud. Gobierno de Chile. Departamento de Estadísticas e Información de Salud, DEIS. Indicadores Básicos de Salud Chile 2016. DEIS, 2016. Disponible en: http://www.deis.cl/wp-content/uploads/2018/12/ IBS-2016.pdf.

20. Chang A, Skirbekk V, Tyrovolas S, Kassebaum N, Dieleman J. Measuring population ageing: an analysis of the Global Burden of Disease Study 2017. Lancet Public Health 2019; 4: e159-67.

21. Ministerio de Salud. Gobierno de Chile. Encuesta Nacional de Salud 2016-2017. Primeros resultados. Disponible en: https://www.minsal.cl/wp-content/uploads/2017/11/ ENS-2016-17_PRIMEROS-RESULTADOS.pdf.

22. Organización Panamericana de la Salud. Organización Mundial de la Salud. Salud en las Américas. Estado de salud de la población. Salud del adulto mayor. Disponible en: https://www.paho.org/salud-en-las-americas-2017/?post_t_es=salud-del-adulto-mayor\&lang=es.

23. World Health Organization. WHO Clinical Consortium on Healthy Ageing. Topic focus: frailty and intrinsic capacity. Report of consortium meeting 1-2 December 2016 in Geneva, Switzerland, WHO, 2017. Disponible en: https://apps.who.int/iris/bitstream/handle/10665/272437/WHO-FWC-ALC-17.2-eng.pdf?ua=1.

24. Ministerio de Desarrollo Social. Segundo Estudio Nacional de la Discapacidad en Chile, 2015. SENADIS, 2016. Disponible en://Users/amleiva/Downloads/Libro\%20 Resultados\%20II\%20Estudio\%20Nacional\%20de \%20 la\%20Discapacidad\%20(1).pdf.

25. Villalobos Dintrans P. Do long-term care services match population needs. A spatial analysis of nursing homes in Chile. PLoS One 2018;13 e0199522.

26. Forttes P, Massad C. Las Personas Mayores en Chile: Situación, avances y desafíos del envejecimiento y la vejez. Servicio Nacional del Adulto Mayor, Santiago de Chile, SENAMA, 2009. Disponible en: http://www.senama.
gob.cl/storage/docs/Las-personas-mayores-de-chile-situacion-avances-desafios-del-envejecimiento-y-vejez-2009.pdf.

27. Fried L, Tangen C, Walson J, Newman A, Hirsch C, Gottdiener J, et al. Frailty in older adults: Evidence for a phenotype. J Gerontol: Med Sci. 2001; 56A (3): M146-M156.

28. Albala C, Lera L, Sánchez H, Angel B, Márquez C, Arroyo $\mathrm{P}$, et al. Frequency of frailty and its association with cognitive status and survival in older Chileans. Clin Interv Aging. 2017; 12: 995-1001.

29. Instituto Nacional de Estadísticas - Chile. Anuario de Estadísticas Vitales, 2017. Período de información: 2017. INE, 2017. Disponible en: https://www.ine.cl/docs/default-source/nacimientos-matrimonios-y-defunciones/ publicaciones-y-anuarios/anuarios-de-estad\%C3\%ADsticas-vitales/anuario-de-estad\%C3\%ADsticas-vitales-017.pdf?sfvrsn=95e68aba_4.

30. OECD. Preventing Ageing Unequally - Action Plan. Meeting of the OECD Council at Ministerial Level, Paris, 2017. Disponible en: https://www.oecd.org/social/CMIN-2017-6-EN.pdf.

31. Anggarawati Putri A, Niken Lestari Ch. The ability to meet the Elderly's basic Needs for Healthy ageing in low-and middle-income countries. ICGH Conference Proceedings. The 1st International Conference on Global Health, 2017.

32. Pan American Health Organization. World Health Organization. Plan of action on the health of the elderly including active and healthy aging. Washington, D.C., USA, 22-26 June 2009.

33. Padilla C, Apablaza M. Caracterización de la calidad de vida En la vejez en chile 1990 y 2015. Universidad del Desarrollo. Centro de Políticas Públicas. Facultad de Gobierno. Documento $N^{\circ} 25,2018$. Disponible en: https://repositorio.udd.cl/bitstream/handle/11447/2135/ An\%C3\%A1lisis-No.-25.pdf? sequence=1\&isAllowe$\mathrm{d}=\mathrm{y}$.

34. Organización Iberoamericana de Seguridad. Situación, necesidades y demandas de las personas mayores en los países del Cono Sur Apuntes para un diagnóstico. Proyecto sobre Personas Mayores, Dependencia y Servicios Sociales en los países del Cono Sur. OISS, 2006. Disponible en: https://oiss.org/wp-content/uploads/2000/01/ Informe_2006_Def_Cono_Sur_-_Apuntes_para_un_ diagnostico_final_-3.pdf.

35. Ministerio de Salud. Gobierno de Chile. Orientación técnica programa más adultos mayores autovalentes. División de Atención Primaria Subsecretaría de Redes Asistenciales. MINSAL, 2015. Disponible en: http://www.bibliotecaminsal.cl/wp/wp-content/ 
uploads/2018/01/013.Orientaci\%C3\%B3n-T\%C3\%A9cnica-Programa-Ms-Autovalentes.pdf.

36. Ministerio de Salud, Gobierno de Chile. Orientación técnica para la atención de salud de las personas adultas mayores en atención primaria. MINSAL, 2013. https:// www.minsal.cl/sites/default/files/files/ot_a_MAYOR. pdf.

37. Ministerio de Salud. Gobierno de Chile. Programa nacional de salud de las personas adultas mayores. Subsecretaria de Salud. Pública División de Prevención y Control de Enfermedades. Departamento de Ciclo Vital. Programa Nacional de Salud del Adulto Mayor. MINSAL, 2014. Disponible en: https://www.minsal.cl/ sites/default/files/files/Borrador\%20documento $\% 20$ Programa\%20Nacional\%20de\%20Personas\%20Adultas\%20Mayores-\%2004-03_14.pdf.

38. Mascayo F, Gajardo J. Ciencia de implementación en la evaluación de programas y servicios de salud en Chile. Rev Med Chile 2018; 146: 942-6.

39. Centro UC Políticas Públicas Chile y sus Mayores.10 años de la Encuesta Calidad de Vida en la Vejez. Resultados IV Encuesta Calidad de Vida en la Vejez. Caja Los Andes, Universidad Católica de Chile, 2017. Disponible en: http://adultomayor.uc.cl/docs/Libro_CHILE_Y_ SUS_MAYORES_2016.pdf.

40. Organización Mundial de la Salud. Estrategia y plan de acción mundiales sobre el envejecimiento y la salud 2016-2020: hacia un mundo en el que todas las personas puedan vivir una vida prolongada y sana. 69a asamblea mundial de la salud Punto 13.4 del orden del día. OMS, 2016. Disponible en: http://apps.who.int/gb/ebwha/ pdf_files/WHA69/A69_ACONF8-sp.pdf.

41. Encuesta Calidad de vida y salud (ENCAVI) 20152016. División de Planificación Sanitaria. Departamento de Epidemiología. Gobierno de Chile. Febrero, 2017. Disponible en: https://www.minsal.cl/wp-content/uploads/2017/02/PRESENTACION-ENCAVI-2016-11.02.2017.pdf.

42. Centro UC Encuestas y Estudios Longitudinales. Evaluación del Pilar Solidario en el financiamiento de la canasta de consumo, los ingresos y la pobreza multidimensional de hombres y mujeres adultos mayores. Informe final, 2017. Disponible en: https://www.previsionsocial.gob.cl/sps/download/estudios-previsionales/ publicaciones/documentos-de-trabajo/2017-2018/ informe-final-pilar-solidario.pdf.

43. Subsecretaria de Previsión Social Gobierno de Chile. Principales resultados de la Encuesta de Calidad de vida del adulto mayor, e impacto del Pilar solidario. Centro Estudios Longitudinales Pontificia Universidad Católica de Chile, CEEL, 2018.
44. Instituto de mayores y Servicios Sociales. La participación Social de las personas mayores. Ministerio de Educación, Política Social y Deporte. Gobierno de España. Madrid, 2008. Disponible en: https://www. imserso.es/InterPresent2/groups/imserso/documents/ binario/11005partsocialmay.pdfBiblioteca del Congreso Nacional de Chile. Programa de política pública, para personas mayores, ejecutados a través de municipalidades. BCN, 2018. Disponible en: https://www. bcn.cl/obtienearchivo?id=repositorio/10221/25810/1/ BCN_Programas_para_adultos_mayores_en_Chile_final.pdf.

45. Gallardo-Peralta L, Conde-Llanes D, Córdova-Jorquera Isabel. Asociación entre envejecimiento exitoso y participación social en personas mayores chilenas. Gerokomos, 2016; 27 (3): 104-8.

46. Arnold M, Herrera F, Massad C, Thumala D. Quinta encuesta nacional de inclusión y exclusión social de las personas mayores en Chile: opiniones de la población chilena respecto al envejecimiento poblacional. Santiago. Ediciones Servicio Nacional del Adulto Mayor, 2018 Disponible en: https://www.academia.edu/37228679/ quinta_encuesta_nacional_inclusi\%c3\%93n_y_exclusi\%c3\%93n_social_de_las_personas_mayores_en_chile_2017_opiniones_de_la_poblaci\%c3\%93n_chilena_respecto_al_envejecimiento.

47. Herrera MS, Kornfeld R, Belloni CL. Trabajo y personas mayores en Chile: Lineamientos para una política de inclusión laboral. Lineamientos para una Política de Inclusión Laboral. Estudio Nacional en personas entre 55 y 74 años. OTIC Del Comercio Servicios y Turismo. Pontificia Universidad Católica de Chile. SENCE, 2018. Disponible en: http://sociologia.uc.cl/wp-content/ uploads/2018/03/trabajo-y-psms-en-chile-web.pdf.

48. Roqué M, Fassio A. Políticas Públicas sobre Envejecimiento en los Países del Cono Sur. Sistema Regional de Información y Aprendizaje para el Diseño de Políticas Públicas en torno al Envejecimiento. SENAMA, 2016. Disponible en: http://www.senama.gob.cl/storage/docs/ Politicas-publicas-vejez-cono-Sur.pdf.

49. Albala C. El envejecimiento de la población chilena y los desafíos para la salud y el bienestar de las personas mayores. 2020. Rev Méd Clín Las Condes, 31 (1): 7-12.

50. Torrado Ramos AM, Sánchez Pérez L, Somonte López R, Cabrera Marsden AM, Henríquez Pino P, Lorenzo Pérez O. Envejecimiento poblacional: una mirada desde los programas y políticas públicas de América Latina, Europa y Asia. Rev. Nov Pob. La Habana. 2014; 10 (19): 18-29.

51. World Health Organization. Active Ageing A Policy Framework. WHO Second United Nations World Assembly 
on Ageing, Madrid, Spain, April 2002. Disponible en: https://apps.who.int/iris/bitstream/handle/10665/67215/ WHO_NMH_NPH_028.pdf?sequence=1

52. Gobierno de Chile. Política Nacional para el Adulto Mayor. Comité Nacional para el Adulto Mayor. Disponible en: https:/www.cepal.org/sites/default/files/events/files/ polnac_chile_0.pdf

53. Ministerio de Desarrollo Social. Gobierno de Chile. Política Integral de Envejecimiento Positivo para Chile 2012-2025. SENAMA, 2016. Disponible en: http:// omayor.cl/wp-content/uploads/2016/05/Pol-tica-Integral-de-Envejecimiento-Activo.pdf

54. Ministerio de Desarrollo Social. Gobierno de Chile. Balance de Gestión Integral Año 2018. SENAMA, 2019. Disponible en: http://www.senama.gob.cl/storage/docs/ BGI2018_V_final.pdf
55. Vergara M, Garay F. Política Integral para la vejez. Informe idea país, mayo 2017. Disponible en: http:// ideapais.cl/wp-content/uploads/2017/11/Politica_Integral_Para_la_Vejez.pdf

56. Huang N-Ch, Chu C, Shiann Far Kung Sh-F, Susan C. $\mathrm{Hu}$ SC. Association of the built environments and health related quality of life in community dwelling older adults: a cross sectional study. Qual Life Res. 2019; 28: 2393-407.

57. Mazacon B, Sánchez J, Sánchez T, Gallegos M Carrion J. Calidad de vida en el Adulto Mayor. Modelo de Atención Integral de Salud. Revista Facultad de Ciencias Médicas. 2018; 21 (1): 20-8.

58. Urzúa A, Bravo M, Ogalde M, Vargas C. Factores vinculados a la calidad de vida en la adultez mayor. Rev Med Chile 2011; 139: 1006-14. 